\title{
Anisotropic Tubular Minimal Path Model with Fast Marching Front Freezing Scheme
}

\author{
Li Liu ${ }^{\mathrm{a}, \mathrm{c}}$, Da Chen ${ }^{\mathrm{b}, \mathrm{c}, \mathrm{d}}$, Laurent D. Cohen ${ }^{\mathrm{c}}$, Jiasong Wu ${ }^{\mathrm{a}}$, Michel Paques ${ }^{\mathrm{d}}$, \\ Huazhong $\mathrm{Shu}^{\mathrm{a}, *}$ \\ ${ }^{a}$ Laboratory of Image Science and Technology, the Key Laboratory of Computer Network \\ and Information Integration, Southeast University,210096 Nanjing, China \\ ${ }^{b}$ Qilu University of Technology (Shandong Academy of Sciences), Shandong Computer \\ Science Center (National Supercomputer Center in Jinan), Shandong Artificial Intelligence \\ Institute, China \\ ${ }^{c}$ Université Paris Dauphine, PSL Research University, CNRS, UMR 7534, CEREMADE, \\ 75016 Paris, France \\ ${ }^{d}$ Centre Hospitalier National d'Ophtalmologie des Quinze-Vingts, Paris, France
}

\begin{abstract}
In this work, we introduce an anisotropic minimal path model based on a new Riemannian tensor integrating the crossing-adaptive anisotropic radius-lifted tensor field and the front freezing indicator by appearance and path features. The non-local path feature only can be obtained during the geodesic distance computation process by the fast marching method. The predefined criterion derived from path feature is able to steer the front evolution by freezing the point causing high bending of the geodesic to solve the shortcut problem. We performed qualitative and quantitative experiments on synthetic and real images (including retinal vessels, rivers and roads) and compare with the minimal path models with classical anisotropic Riemannian metric and dynamic isotropic metric, which demonstrated the proposed method can detect desired targets from complex tubular tree structures.
\end{abstract}

Keywords: Minimal path model, Anisotropy enhancement, Riemannian metric, Path feature, Tubular structures

\footnotetext{
* Corresponding author

Email address: shu.list@seu.edu.cn (Huazhong Shu)
} 


\section{Introduction}

Tubular structure extraction is a crucial task in many computer vision applications. Various tubular structure extraction algorithms and techniques have been studied, which can be roughly divided into two categories: supervised and 5 unsupervised methods $[1,2]$. Supervised approaches usually achieve better performance due to the prior information from the training components to decide whether a pixel should belong to a vessel or not. These models construct feature vectors in order to train the respective classifiers $[3,4]$ or apply the neural network $[5,6]$ with the help of manual label. However, it is very time-consuming to find discrimination features for constructing powerful models by training. Besides, the obtained models always meet for particular requirement. Those unsupervised methods, without manual labeling information, usually have lower computation complexity and more simple procedure [2], such as the pattern recognition techniques [7, 8], model-based approaches [9, 10], tracking-based algorithms $[11,12]$ and so on.

The minimal path technique [13] has been adopted to detect tubular structures thanks to its efficiency and global optimality $[14,15,16,17]$. It is often taken as an interactive tool such that users are allowed to provide manual intervention such as source points, which constrains the minimal path to delineate the tubular structures. In essence, a geodesic path is a curve linking two points derived by globally minimizing a weighted curve length [13]. It is defined by integrating a geodesic metric $\mathcal{P}: \Omega \times \mathbb{R}^{d} \rightarrow \mathbb{R}^{+}$, where $\Omega \subset \mathbb{R}^{d}$ stands for the open and bounded domain and $d$ is the dimension of $\Omega$, along a regular path $\gamma$ :

$$
\mathfrak{L}(\gamma)=\int_{0}^{1} \mathcal{P}\left(\gamma(t), \gamma^{\prime}(t)\right) \mathrm{d} t
$$

where $\gamma:[0,1] \rightarrow \Omega \subset \mathbb{R}^{d}$ is a regular curve of Lipschitz continuity. The minimal path models have been extensively exploited for tubular structure segmentation, where both the centerline positions and thickness of the tubular structures are required $[15,16,17]$. The isotropic tubular minimal path model [15] only makes use of the curve position information, which may increase the risk of shortcuts. 
More general anisotropic Riemannian metric $[16,18]$ is proposed to segment tubular structures in conjunction of the pre-detected vessel anisotropy features. However, this extended tubular minimal path model still has difficulties when dealing with complicated situation such as extract a weak vessel from a tree structure. Moreover, some researchers try to improve the classical tubular minimal path models by reducing the user intervention [19, 20, 21]. For these models, only one (or several) initial root point(s) is (are) necessary to extract a tubular tree structure. Recently, the higher order properties of the geodesic curves are exploited for shortest path estimation as well as tubular structure segmentation. Specifically, Ulen et al. [22] proposed a curvature and torsion regularized shortest path model by computing the curvature and the torsion. Based on the Eikonal equation framework, the curvature-penalized minimal path models $[23,24,25]$ are established over an orientation-lifted space such that the orientation dimension can be used to represent the curve tangents.

Controlling the course of the fast marching front propagation is a solution for the above problem. Criterions are defined to make the front propagation stop in undesired directions or promote in specific directions [26, 27]. In contrast to the minimal path models using static geodesic metrics which are fixed during fast marching front propagation, the dynamic minimal path models allow to update 40 the respective geodesic metrics in the course of geodesic distance estimation. In [28], the authors proposed a new fast marching front propagation scheme in order to address the shortcut and short branches combination problems. The basic idea is to freeze the front points of which the respective local path features violate the pre-defined criteria. In this case the fast marching fronts will not pass through the points corresponding to undesired geodesic paths. Chen et al. [29] proposed an adaptively Riemannian metric using a dynamic metric construction way in order to penalize the appearance feature coherence property. In this case the obtained geodesic paths favour to pass by the region of slow-varying tubular features. Geoffrey et al. [30] created a connectivity metric to determine the reasonable pathways of connection. Windheuser et al. [31] estimated the curvature information by computing the angles between each pair of adjacent 


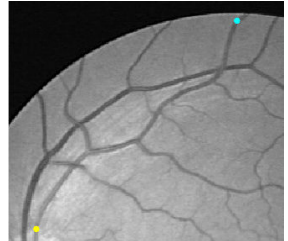

(a)

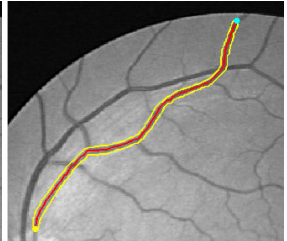

(b)

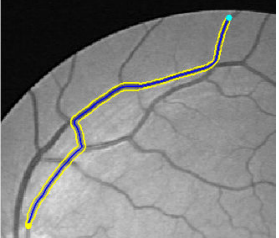

(c)

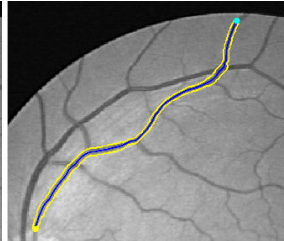

(d)

Figure 1: (a)The yellow and cyan dots incidate the source and end points. (b) The red dash line indicates the target tubular structure centerline. (c) The geodesic path (blue line) obtained by the anisotropic tubular minimal path model [16], where the short branches combination problem occurs. (d) The geodesic path (blue line) derived from the proposed model. (Yellow lines denote the vessel boundaries)

edges. Krueger et al. [32] incorporated the curvature estimation into a greedy core algorithm to improve the efficiency. However, these models track geodesic paths only in the spatial space where the vessel thickness information cannot be extracted.

In this paper, we focus on the dynamic minimal path-based tubular structure segmentation, for which the geodesic metrics are established over a radius-lifted space. The existing tubular minimal path models $[15,16,17]$ often suffer from the shortcut and short branches combination problems, especially for the case that the target is weakly-defined and crosses or parallels with strong ones, see Fig. 1. In this figure, the target vessel has low gray level contrast, while its neighbour vessels are strongly-defined (i.e., high gray level contrast). In Fig. 1(c), we show the minimal path extraction result obtained by the anisotropic tubular minimal path model [16]. One can observe short branches combination occur. In other words, the obtained minimal path prefers to pass through strong segments belonging to different vessels. In order to simultaneously obtain the centerlines and thickness of tubular structures as well as to overcome the shortcut and short branches combination problems, we proposed an anisotropic minimal path model for tubular structure segmentation via a dynamic radius-lifted anisotropic metric and state-of-the-art anisotropic fast marching method [33]. The motivation of this work is that the anisotropic tubular minimal path model 
[16] cannot take into account path features for distance estimation. Using the path features detected during the front propagation for minimal path computation is able to obtain desired results even in complicated situation. The main contribution in this paper lies at the introduction of a dynamic anisotropic Riemannian metric in the radius-lifted space using back-tracked geodesic paths. In Fig. 1(d), we present the result obtained by the proposed model. One can point out that the blue line indeed correctly indicates the target.

The paper outline is shown as follows. In Section 2, we briefly introduce the background for the anisotropic radius-lifted Riemannian metric. Section 3 describes the main contribution of this work including the proposed dynamic Riemannian metric in a radius-lifted space and its numerical implementation in conjunction with the fast marching method. Section 4 shows the experimental results and the final section 5 gives the conclusion.

\section{${ }_{85}$ 2. Minimal Paths for Tubular Structure Segmentation}

In this section, we give the background on anisotropic radius-lifted minimal path model [16] for tubular structure segmentation.

\subsection{Tubular Geometry Features Computation}

In this section, we first consider the extraction of the local tubular geometry features from the image $I: \Omega \rightarrow \mathbb{R}$ through the optimally oriented flux (OOF) filter [34]. Note that the local geometry features can also be extracted using other steerable filters such as $[34,35,36]$. The output of the OOF filter is established in a multi-scale space defined as $\hat{\Omega}:=\Omega \times\left[r_{\min }, r_{\max }\right]$, where $\left[r_{\min }, r_{\max }\right]$ is the radius space ${ }^{1}$. The output of the OOF filter at the position $\mathbf{x}$ and scale $r$ is a symmetric matrix of size $d \times d$ as follows:

$$
\mathbf{F}(\mathbf{x}, r)=\frac{1}{r}\left(\left(\partial_{i, j} G_{\sigma}\right) * I * \chi_{S_{r}}\right)(\mathbf{x}, r),
$$

\footnotetext{
${ }^{1}$ In the space $\hat{\Omega}$, a point $\hat{\mathbf{x}} \in \hat{\Omega}$ is an ordered pair $(\mathbf{x}, r)$ where $\mathbf{x} \in \Omega \subset \mathbb{R}^{2}$ and $r \in$ $\left[r_{\min }, r_{\max }\right]$.
} 
where $\left(\partial_{i, j} G_{\sigma}\right)$ is the Hessian matrix of the Gaussian kernel $G_{\sigma}$ with variance $\sigma . \chi_{S_{r}}$ is a characteristic function of a disk with radius $r$.

The eigenvalues $\lambda_{i}(i=1,2, \cdots, d)$ extracted using the OOF filter are the values of the oriented flux along the corresponding eigenvectors $\mathbf{v}_{i}$ as following:

$$
\lambda_{i}(\mathbf{x}, r)=\mathbf{v}_{i}^{T}(\mathbf{x}, r) \mathbf{F}(\mathbf{x}, r) \mathbf{v}_{i}(\mathbf{x}, r) .
$$

Without loss of generality, we assume that $\lambda_{1}(\cdot) \leq \lambda_{2}(\cdot) \leq \cdots \lambda_{d}(\cdot)$. In this paper, we only consider the $2 \mathrm{D}$ tubular segmentation problem, which means that $d=2$. For the case that the tubular structures have lower gray levels than background, the vector $\mathbf{v}_{1}\left(\mathbf{x}, r^{*}\right)$ indicates the orientation of the tubular structure at the point $\mathbf{x}$ inside the vessel structures, where $r^{*}$ is the optimal vessel scale of point $\mathbf{x}$.

\subsection{Radius-lifted Minimal Paths for Tubular Segmentation}

We denote by $\mathcal{S}_{d}^{+}$the set of symmetric positive definite matrices of size $d \times d(d=2,3)$ and let $\operatorname{Lip}([0,1], \hat{\Omega})$ be the set of Lipchitz continuous curves ${ }_{100} \gamma:[0,1] \rightarrow \hat{\Omega}$. The radius-lifted minimal path model is proposed in [15] for tubularity segmentation. Benmannsour and Cohen [16] generalize this isotropic model [15] to the anisotropic case. The basic idea for both models is to lift a spatial geodesic to the radius-lifted space $\hat{\Omega}$ by adding one abstract dimension to represent the vessel radii.

The path length $\mathfrak{L}$ for such a radius-lifted curve $\gamma \in \operatorname{Lip}([0,1], \hat{\Omega})$ can be measured through a Riemannian tensor field $\mathcal{M}_{\text {scale }}: \hat{\Omega} \rightarrow S_{3}^{+}$,

$$
\mathfrak{L}(\gamma)=\int_{0}^{1} \sqrt{\left\langle\gamma^{\prime}(u), \mathcal{M}_{\text {scale }}(\gamma(u)) \gamma^{\prime}(u)\right\rangle} d u,
$$

where $\langle\cdot, \cdot\rangle$ represents the Euclidean scalar product. The tensor field $\mathcal{M}_{\text {scale }}$ is constructed [16] by the eigenvalues $\lambda_{i}$ and eigenvectors $\mathbf{v}_{i}$,

$$
\mathcal{M}_{\text {scale }}(\mathbf{x}, r)=\left(\begin{array}{cc}
\mathcal{M}_{\text {aniso }}(\mathbf{x}, r) & \mathbf{0} \\
\mathbf{0} & \mathcal{P}_{\text {scale }}(\mathbf{x}, r)
\end{array}\right),
$$

where $\mathcal{M}_{\text {aniso }}: \hat{\Omega} \rightarrow S_{2}^{+}$is a tensor field associated to the spatial anisotropy with size $2 \times 2$ and $\mathcal{P}_{\text {scale }}: \hat{\Omega} \rightarrow \mathbb{R}^{+}$is a scalar function. Both can be constructed 
by combining the eigenvalues and eigenvectors of the OOF response $\mathbf{F}$ in Eq. (2) as follows:

$$
\begin{aligned}
& \mathcal{M}_{\text {aniso }}(\hat{\mathbf{x}})=\exp \left(\alpha \lambda_{1}(\hat{\mathbf{x}})\right) \mathbf{v}_{2}(\hat{\mathbf{x}}) \mathbf{v}_{2}^{T}(\hat{\mathbf{x}})+\exp \left(\alpha \lambda_{2}(\hat{\mathbf{x}})\right) \mathbf{v}_{1}(\hat{\mathbf{x}}) \mathbf{v}_{1}^{T}(\hat{\mathbf{x}}) \\
& \mathcal{P}_{\text {scale }}(\hat{\mathbf{x}})=\beta \exp \left(\alpha \lambda_{2}(\hat{\mathbf{x}})\right)
\end{aligned}
$$

105

where $\alpha \in \mathbb{R}$ and $\beta \in \mathbb{R}^{+}$are two parameters which control the regularization of the spatial and radius dimensions, respectively. Note that the value of $\alpha$ is negative for dark-vessel and bright-background case and $\alpha>0$, otherwise.

In the Riemannian minimal path model, once the metric tensor is given, one can define the relevant geodesic distance $\mathcal{U}_{\hat{\mathbf{s}}}(\hat{\mathbf{x}})$ as the minimal curve length between $\hat{\mathbf{x}}$ and the fixed source point $\hat{\mathbf{s}}$ as follows

$$
\mathcal{U}_{\hat{\mathbf{s}}}(\hat{\mathbf{x}})=\inf _{\gamma \in \operatorname{Lip}([0,1], \hat{\Omega})}\{\mathfrak{L}(\gamma) ; \gamma(0)=\hat{\mathbf{s}}, \gamma(1)=\hat{\mathbf{x}}\},
$$

where the values of $\mathcal{U}_{\hat{\mathbf{s}}}$ can be regarded as the arrival times of a front propagating with oriented velocity related to the metric tensor $\mathcal{M}_{\text {scale }}^{-1}$. The geodesic distance map $\mathcal{U}_{\hat{\mathbf{s}}}$ from the source point $\hat{\mathbf{s}}$ satisfies the anisotropic Riemannian Eikonal equation

$$
\left\|\nabla \mathcal{U}_{\hat{\mathbf{s}}}(\hat{\mathbf{x}})\right\|_{\mathcal{M}_{\text {scale }}^{-1}(\hat{\mathbf{x}})}=1, \quad \forall \hat{\mathbf{x}} \in \hat{\Omega} \backslash\{\hat{\mathbf{s}}\},
$$

with boundary condition $\mathcal{U}_{\hat{\mathbf{s}}}(\hat{\mathbf{s}})=0$, where $\|\mathbf{u}\|_{M}=\sqrt{\mathbf{u}^{T} M \mathbf{u}}$ for any matrix $M \in S_{3}^{+}$.

A geodesic path $\hat{\mathcal{C}}_{\hat{\mathbf{x}}, \hat{\mathbf{s}}}$ linking $\hat{\mathbf{x}}$ to the source point $\hat{\mathbf{s}}$ can be tracked from the point $\hat{\mathbf{x}}$ by solving the following ordinary differential equation (ODE) till the source point $\hat{\mathbf{s}}$ is reached

$$
\tilde{\mathcal{C}}_{\hat{\mathbf{x}}, \hat{\mathbf{s}}}^{\prime}(s)=-\frac{\mathcal{M}_{\text {scale }}^{-1}\left(\tilde{\mathcal{C}}_{\hat{\mathbf{x}}, \hat{\mathbf{s}}}(s)\right) \nabla \mathcal{U}_{\hat{\mathbf{s}}}\left(\tilde{\mathcal{C}}_{\hat{\mathbf{x}}, \hat{\mathbf{s}}}(s)\right)}{\left\|\mathcal{M}_{\text {scale }}^{-1}\left(\tilde{\mathcal{C}}_{\hat{\mathbf{x}}, \hat{\mathbf{s}}}(s)\right) \nabla \mathcal{U}_{\hat{\mathbf{s}}}\left(\tilde{\mathcal{C}}_{\hat{\mathbf{x}}, \hat{\mathbf{s}}}(s)\right)\right\|}
$$

with $\tilde{\mathcal{C}}_{\hat{\mathbf{x}}, \hat{\mathbf{s}}}(0)=\hat{\mathbf{x}}$. The geodesic $\tilde{\mathcal{C}}_{\hat{\mathbf{x}}, \hat{\mathbf{s}}}$ is parameterized by its arc-length. The final geodesic $\mathcal{C}_{\hat{\mathbf{s}}, \hat{\mathbf{x}}} \in \operatorname{Lip}([0,1], \Omega)$ joining from the source point $\hat{\mathbf{s}}$ to $\hat{\mathbf{x}}$ can be obtained by reversing and reparameterizing $\tilde{\mathcal{C}}_{\hat{\mathbf{x}}, \hat{\mathbf{s}}}$. 


\section{The New Anisotropic Geodesic Metric with Nonlocal Information in Radius-lifted Space}

Overview. The main goal in this section is to establish a new anisotropic geodesic metric in a radius-lifted space in order to avoid the shortcut and short branches combination problems as shown in Fig. 1. In contrast to [16] for which only the local geometry information are considered to establish the geodesic metrics, the front freezing-based minimal path model [28] constructs the geodesic metrics by taking into account the nonlocal information such as the local path. During the geodesic distance front propagation, the front points for which the features do not satisfy the given criterion will be frozen, where the features for each front point are extracted through two extra points. This scheme proposed in [28] is able to avoid the shortcut problem in some extent. However, it is restricted to the case of centerline detection and cannot take advantages of the path orientation due to the isotropic nature of the used metrics in [28]. In this section, we extend this front frozen scheme to a radius-lifted space through an anisotropic Riemannian metric. This is done by constructing a new Riemannian tensor field $\mathcal{M}_{\mathrm{dyn}}: \hat{\Omega} \rightarrow S_{3}^{+}$during the geodesic distance computation which is actually carried out in a front advancing procedure. The invoked tensor field $\mathcal{M}_{\text {dyn }}$ consists of two ingredients: the crossing-adaptive anisotropic radius-lifted tensor field $\mathcal{M}_{\text {adap }}: \hat{\Omega} \rightarrow S_{3}^{+}$and the front freezing indicator $\delta: \hat{\Omega} \rightarrow\{1, \infty\}$. It can be expressed by

$$
\mathcal{M}_{\text {dyn }}(\mathbf{x}, r)=\mathcal{M}_{\text {adap }}(\mathbf{x}, r) \times \delta(\mathbf{x}, r), \forall(\mathbf{x}, r) \in \hat{\Omega} .
$$

We will explain the construction details for the tensor field $\mathcal{M}_{\text {adap }}$ in Section 3.1 and the scalar indicator $\delta$ in Section 3.2, respectively.

\subsection{Computation of the Crossing-Adaptive Tensor Field}

By applying the OOF filter to an image involving vessel tree structures, one can obtain an optimal direction $\mathbf{v}_{1}(\mathbf{x}, r)$ at each point $(\mathbf{x}, r)$ belonging to the 


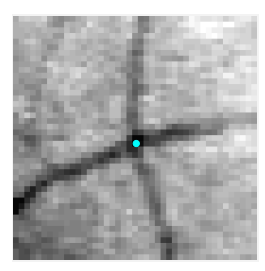

(a)

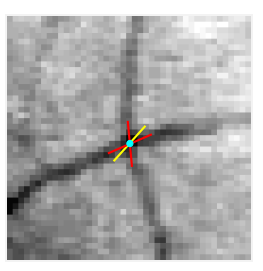

(b)

Figure 2: (a) A retina image with a crossing point denoted as cyan dot. (b) The red curves illustrate the vessel direction passing through the crossing point. Yellow curve denotes the vessel direction detected by the OOF filter.

tree structures through eigen-decomposition to the response of the filter OOF

$$
\mathbf{F}(\mathbf{x}, r)=\sum_{i=1}^{2} \lambda_{i}(\mathbf{x}, r) \mathbf{v}_{i}(\mathbf{x}, r), \quad \text { s.t. } \quad \lambda_{1}(\cdot) \leq \lambda_{2}(\cdot)
$$

We suppose that inside the tubular structures the intensities are lower than background, thus the corresponding eigenvalue $\lambda_{2}$ can be used to characterize the appearance feature of the vessels, by which we define a vesselness map $\zeta: \Omega \rightarrow \mathbb{R}_{0}^{+}$

$$
\zeta(\mathbf{x})=\max \left\{\max _{r} \lambda_{2}(\mathbf{x}, r), 0\right\} .
$$

The value of $\zeta(\mathbf{x})$ is derived from $\lambda_{2}$ at the optimal scale $\eta(\mathbf{x}) \in\left[r_{\min }, r_{\max }\right]$, where the $\eta: \Omega \rightarrow\left[r_{\min }, r_{\max }\right]$ is a map defined by

$$
\eta(\mathbf{x})=\underset{r \in\left[r_{\min }, r_{\max }\right]}{\arg \max }\left\{\lambda_{2}(\mathbf{x}, r)\right\}
$$

Based on the optimal scale map $\eta$ and the eigenvectors $\mathbf{v}_{1}$, we can define the tubular feature vector $\mathfrak{p}: \Omega \rightarrow \mathbb{R}^{2}$ that indicates the orientation for a tubular structure

$$
\mathfrak{p}(\mathbf{x})=\mathbf{v}_{1}(\mathbf{x}, \eta(\mathbf{x})) .
$$

The tubular direction $\mathfrak{p}(\mathbf{x})$ well describes the vessel orientation at non-crossing point $\mathbf{x}$. However, for crossing points, denoted by $\mathbf{y}$, there will be at least two vessels across one another. This means that the highly anisotropic tensors $\mathcal{M}_{\text {aniso }}(\mathbf{y}, \cdot)$ defined in Eq. (5) may lead to incorrect geodesic tangents at these 


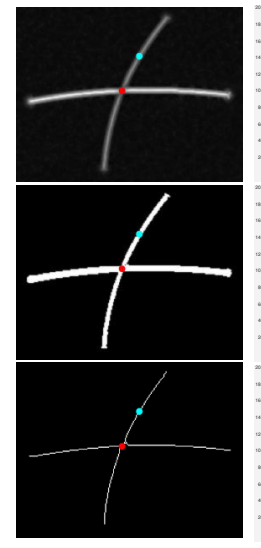

(a)

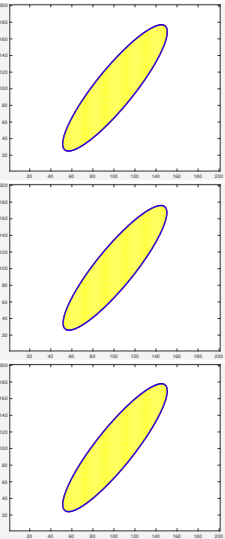

(b)

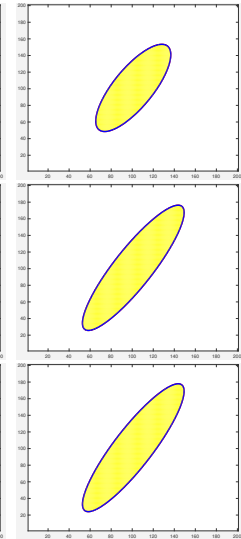

(c)

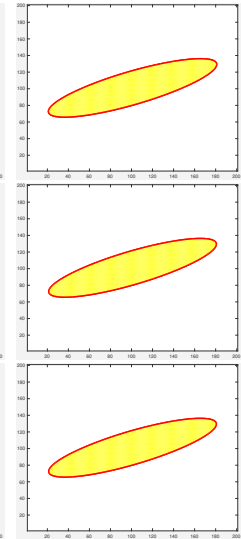

(d)

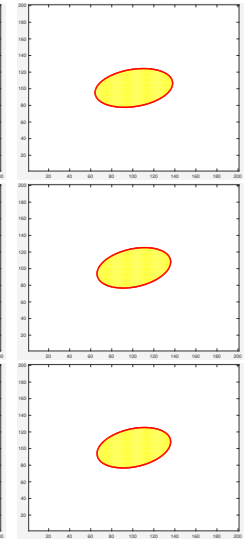

(e)

Figure 3: (a) A crossing structure with two sampled points. Columns (b) and (c): The unit balls for the proposed $\mathcal{M}_{\text {adap }}$ with $p=1$ and $p=7$ at the single vessel point denoted as cyan dot. Columns (d) and (e): The unit balls for the proposed $\mathcal{M}_{\text {adap }}$ with $p=1$ and $p=7$ at the crossing point displayed as a red dot.

crossing points y. An example is given in Fig. 2, where the red lines indicate the true vessel directions through a crossing point denoted by a cyan dot. The yellow line indicates the vessel direction detected by the OOF filter, however it is incorrect. This may lead to the wrong vessel segmentation. A solution to this problem is to adaptively remove the anisotropy from the tensors $\mathcal{M}_{\text {aniso }}(\mathbf{y}, \cdot)$, which can be done by invoking the orientation scores [2] or by structure tensors [37]. In this section, we construct our crossing-adaptive radius-lifted tensor field through the tool of structure tensor.

The shortcut and short branches combination problems usually occur where the vessel crosses its neighbours, since the eigenvector of a crossing point usually indicates the orientation of the vessel with strong appearance feature. So the speed computed from the anisotropic geodesic metric is slower along the weak vessel direction than along the strong one. To solve the shortcut and short branches combination problems, we make use of a crossing-adaptive tensor field $\mathcal{M}_{\text {adap }}: \hat{\Omega} \rightarrow S_{3}^{+}$established in the radius-lifted space $\hat{\Omega}$, the anisotropy of which is kept at single-vessel region and removed or reduced at crossing points. 
It can be constructed by

$$
\mathcal{M}_{\text {adap }}(\mathbf{x}, r)=\operatorname{diag}\left(\exp \left(-\alpha \lambda_{2}(\mathbf{x}, r)\right) \mathcal{T}_{\text {smooth }}(\mathbf{x}, r), \mathcal{P}_{\text {scale }}(\mathbf{x}, r)\right)
$$

where $\alpha$ is a positive constant which has been used in Eqs. (6) and (7). The tensor field $\mathcal{T}_{\text {smooth }}: \hat{\Omega} \rightarrow S_{2}^{+}$is computed via a Gaussian kernel $G_{p}$ with standard derivative $p$ and the identity matrix $\mathrm{I}_{\mathrm{d}}$ of size $2 \times 2$

$$
\mathcal{T}_{\text {smooth }}(\mathbf{x}, \cdot)=\left(\frac{\left(G_{p} * \mathcal{T}\right)(\mathbf{x})}{\left(G_{p} * \hbar\right)(\mathbf{x})}+\epsilon \mathrm{I}_{\mathrm{d}}\right)^{-1}, \quad \mathcal{T}(\mathbf{x})=\hbar(\mathbf{x}) \mathfrak{p}(\mathbf{x}) \mathfrak{p}^{T}(\mathbf{x})
$$

where $\epsilon \in \mathbb{R}^{+}$is a sufficiently small constant to generate a regular matrix $\mathcal{M}_{\text {adap }}(\cdot)$. Note that for any radius values $r_{1}, r_{2} \in\left[r_{\min }, r_{\max }\right]$ where $r_{1} \neq$ $r_{2}$, one always has $\mathcal{T}_{\text {smooth }}\left(\mathbf{x}, r_{1}\right)=\mathcal{T}_{\text {smooth }}\left(\mathbf{x}, r_{2}\right)$. The scalar-valued function $\hbar: \Omega \rightarrow \mathbb{R}_{0}^{+}$is a weighted function over the image domain $\Omega$. In this paper, we propose three ways to compute the function $\hbar$, where the first way is to invoke the vesselness map $\zeta$ (see Eq. (13)).

$$
\hbar(\mathbf{x})=\zeta(\mathbf{x}), \quad \forall \mathbf{x} \in \Omega
$$

Alternatively, the weighted function $\hbar$ can be computed as a binary-valued function, which depends on a threshold value $\mathrm{Th} \in \mathbb{R}^{+}$and also on the vesselness $\operatorname{map} \zeta$

$$
\hbar(\mathbf{x})= \begin{cases}1, & \text { if } \zeta(\mathbf{x})>\mathrm{Th} \\ 0, & \text { otherwise }\end{cases}
$$

The last method utilizes the skeleton of the vessel mask. The function $\hbar$ is used in order to reduce the influence from the regions outside the vessel structures. In other words, the information inside the vessel region dominates the tensor field $\mathcal{T}_{\text {smooth }}$.

In Eq. (18), the function $\hbar$ is set to be the vesselness map which has small values outside the vessel regions but large values inside, where an example can be found in the first row of column (a) of Fig. 3. This is an adaptive way for the computation of the tensor field $\mathcal{T}_{\text {smooth }}$ comparing to the other two ways which set $\hbar$ as a binary-valued function. Considering the vessel segmentation 
has been studied for a long time, the vessel mask is easy to be computed by many existing approaches and the skeleton can be obtained from the vessel mask. For the second method expressed in Eq. (19), a point $\mathbf{x}$ for which $\hbar(\mathbf{x})=1$ implies that $\mathbf{x}$ is located inside a vessel. An example is shown in the second row of column (a) in Fig. 3. In the third method, $\hbar(\mathbf{x})=1$ means that $\mathbf{x}$ is a centreline point, see the third row of column (a) of Fig. 3 for an example. In this case, the information on the centreline positions dominates the tensor field $\mathcal{T}_{\text {smooth }}$. For the crossing point, the tensor field $\mathcal{T}_{\text {smooth }}$ is impacted greater by the vessel with bigger radius. Note that the main difference between $\mathcal{T}_{\text {smooth }}$ and the structure tensor field used in [37] lies at the existence of the function $\hbar$. As discussed above, it can reduce or avoid the effects derived from the non-vessel regions. This means that we are able to make use of a Gaussian filter with more flexible variance values $p$.

We express the tensor filed $\mathcal{T}_{\text {smooth }}^{-1}$ by

$$
\mathcal{T}_{\text {smooth }}^{-1}(\mathbf{x}, \cdot)=\int_{\Omega} \frac{G_{p}(\mathbf{y}) \hbar(\mathbf{x}-\mathbf{y}) \mathfrak{p}(\mathbf{x}-\mathbf{y}) \mathfrak{p}^{T}(\mathbf{x}-\mathbf{y}) d \mathbf{y}}{G_{p}(\mathbf{y}) \hbar(\mathbf{x}-\mathbf{y}) d \mathbf{y}}+\epsilon \mathrm{I}_{\mathrm{d}}
$$

One can see that the Gaussian filtering is operated on the feature vectors $\mathfrak{p}(\mathbf{y})$ (indicating the vessel orientations) at the vicinity of a point $\mathbf{x}$. If $\mathbf{x}$ is a vessel segment point and the vectors $\mathfrak{p}(\mathbf{y})$ change slowly, then the eigenvector of the tensor $\left(G_{p} * \mathcal{T}\right)(\mathbf{x})$, which corresponds to the largest eigenvalue, will approximate to the feature vector $\mathfrak{p}(\mathbf{x})$. For the vessel points $\mathbf{x}$ nearby a crossing structure, the feature vectors potentially vary fast, leading to the tensors $\left(G_{p} * \mathcal{T}\right)(\mathbf{x})$ which are nearly isotropic. As shown in Fig. 3, the anisotropy of the metric at point (e.g. the red point) located on the crossing section is removed apparently by the Gaussian filtering, however, the anisotropy of the metric of the single vessel point (e.g. cyan point) is contained adaptively.

\subsection{Computation of the Front-Freezing Indicator}

In this section, we construct the proposed front-freezing indicator $\delta$ with non-local path features derived from the local truncated geodesic paths to solve the shortcut and short branches combination problems. The non-local feature is 


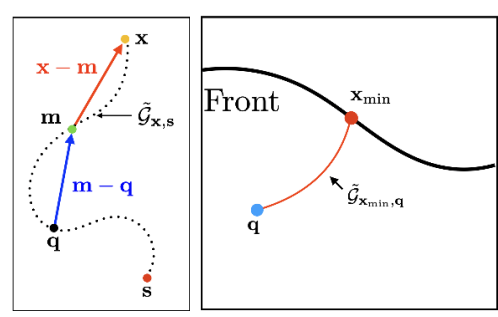

(a) (b)

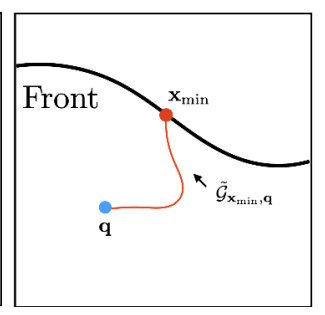

(c)

Figure 4: (a) The extra points $\mathbf{q}$ and $\mathbf{m}$ identified from the projected geodesic path $\tilde{\mathcal{G}}_{\mathbf{x}, \mathbf{s}}$. (b) and (c) Projected path $\tilde{\mathcal{G}}_{\hat{\mathbf{x}}_{\text {min }}, \mathbf{q}}$ (red lines) with small and large bending measures, respectively. The red dot in figure (b) (or figure (c)) indicates the spatial component $\mathbf{x}_{\min }$ of the latest Trial point $\hat{\mathbf{x}}_{\min }=\left(\mathbf{x}_{\min }, r_{\min }\right)$.

described by the bending measure of the local path computed through two extra points located on it. Moreover, we first present the principle for the construction of the indicator $\delta$ and the numerical implementation method based on the fast marching method will be described in Section 3.3.

Here we give the method to identify the extra two points on a local geodesic path linking a target point $\hat{\mathbf{x}}=\left(\mathbf{x}, r_{\mathbf{x}}\right)$ to the source point $\hat{\mathbf{s}}=\left(\mathbf{s}, r_{\mathbf{s}}\right)$. First of all, the geodesic path can be computed by solving the gradient descent ODE (see in Eq. (10)) on the obtained geodesic distance map $\mathcal{U}_{\hat{\mathbf{s}}}$, which has already been computed within the region where the front visited [28]. During the front propagation, one can easily track a radius-lifted geodesic path $\tilde{\mathcal{C}}_{\hat{\mathbf{x}}, \hat{\mathbf{s}}}$ parameterized by its arc-length by solving the corresponding gradient descent ODE (10). Since $\tilde{\mathcal{C}}_{\hat{\mathbf{x}}, \hat{\mathbf{s}}}$ lies in the radius-lifted space, we have $\tilde{\mathcal{C}}_{\hat{\mathbf{x}}, \hat{\mathbf{s}}}=\left(\tilde{\mathcal{G}}_{\mathbf{x}, \mathbf{s}}, \eta\right)$ where $\tilde{\mathcal{G}}_{\mathbf{x}, \mathbf{s}}(\cdot) \in \Omega$ is the projected path and $\eta(\cdot) \in\left[r_{\min }, r_{\max }\right]$. We first detect a point $\hat{\mathbf{q}}=\left(\mathbf{q}, r_{\mathbf{q}}\right)$ from $\tilde{\mathcal{C}}_{\hat{\mathbf{x}}, \mathbf{s}}$ such that its projected path $\left|\mathcal{G}_{\mathbf{x}, \mathbf{q}}\right|=\Gamma$, where $\left|\mathcal{G}_{\mathbf{x}, \mathbf{q}}\right|$ stands for the Euclidean length of $\mathcal{G}_{\mathbf{x}, \mathbf{q}}$ and $\Gamma$ is a positive constant. Note that $\hat{\mathbf{q}}$ is the first extra point and the second one, referred to as $\hat{\mathbf{m}}=\left(\mathbf{m}, r_{\mathbf{m}}\right)$, is defined as the middle point of the part of the path $\tilde{\mathcal{C}}_{\hat{\mathbf{x}}, \hat{\mathbf{q}}}$. The process to search the two extra points are shown in Fig. $4(\mathrm{a})$. The bending measure $\mathcal{K}$ is estimated by the angle 
between two vectors related to the two extra points $\mathbf{q}, \mathbf{m} \in \Omega$ as follows [28]

$$
\mathcal{K}(\mathbf{x}, r)=\frac{\langle\mathbf{m}-\mathbf{q}, \mathbf{x}-\mathbf{m}\rangle}{\|\mathbf{m}-\mathbf{q}\|\|\mathbf{x}-\mathbf{m}\|},
$$

The values of $\mathcal{K}$ are ranged at $[-1,1]$. The estimation of $\mathcal{K}$ is done during the fast marching front propagation, which will be described in Section 3.3. We mention that a small value of $\mathcal{K}$ implies that the corresponding geodesic path likely appears with a sharp turning.

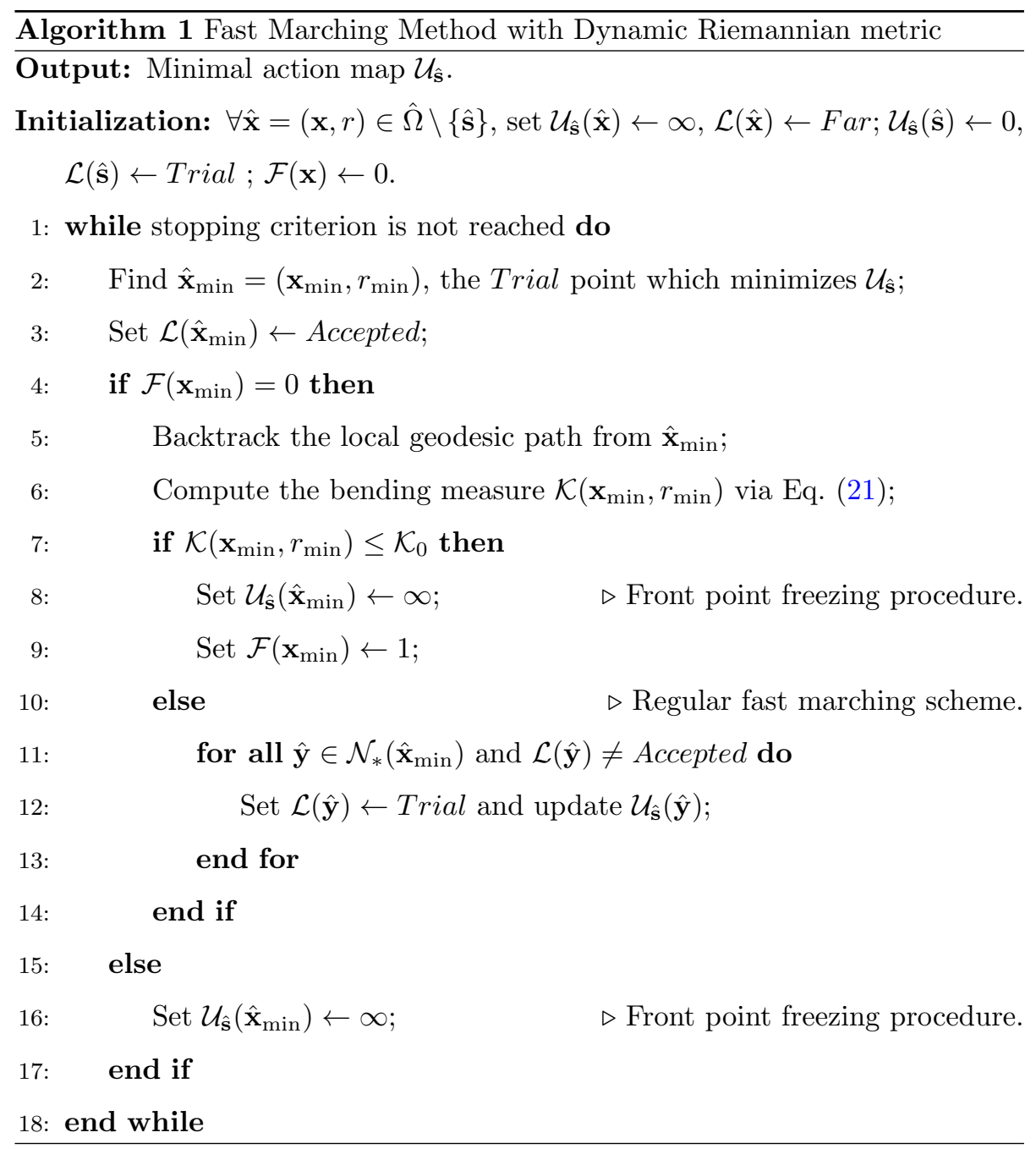


Now we give the computation method for the front-freezing indicator $\delta$ : $\hat{\Omega} \rightarrow\{1, \infty\}$, which takes into account the non-local feature to freeze the front points causing a geodesic path of high values of $\mathcal{K}$. The criterion is based on the fact that the orientations of the tubular structure such as blood vessels usually varies smoothly without sharp turning. The indicator $\delta$ is constructed to determine the bending range of the local tubular structure:

$$
\delta(\hat{\mathbf{x}})= \begin{cases}1 & \mathcal{K}(\hat{\mathbf{x}})>\mathcal{K}_{0} \\ +\infty & \text { otherwise }\end{cases}
$$

175 front is comprised of all the grid points tagged as Trial. In each geodesic distance updating step, we apply the method presented in Section 3.2 by setting that $\hat{\mathbf{x}}:=\hat{\mathbf{x}}_{\min }$ to compute the value of the indicator $\mathcal{K}$ for the latest Trial point $^{2} \hat{\mathbf{x}}_{\text {min }}$

\footnotetext{
${ }^{2}$ the point of smallest geodesic distance value among all the Trial points
} 


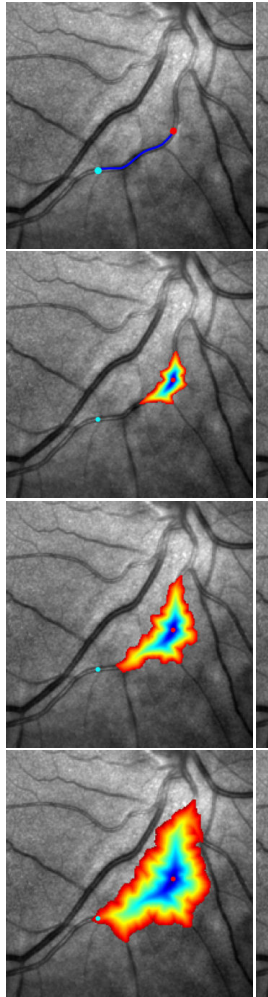

(a) $\operatorname{ArR}$

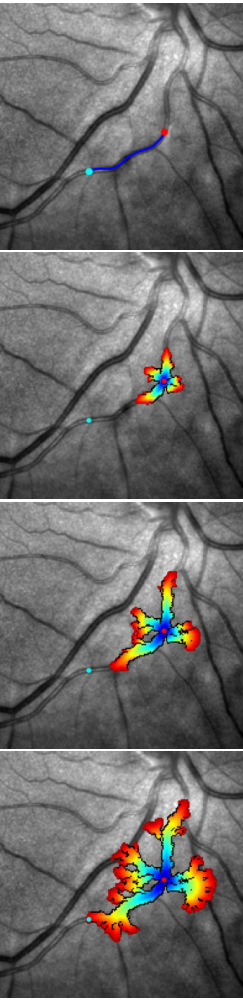

(b) dArR

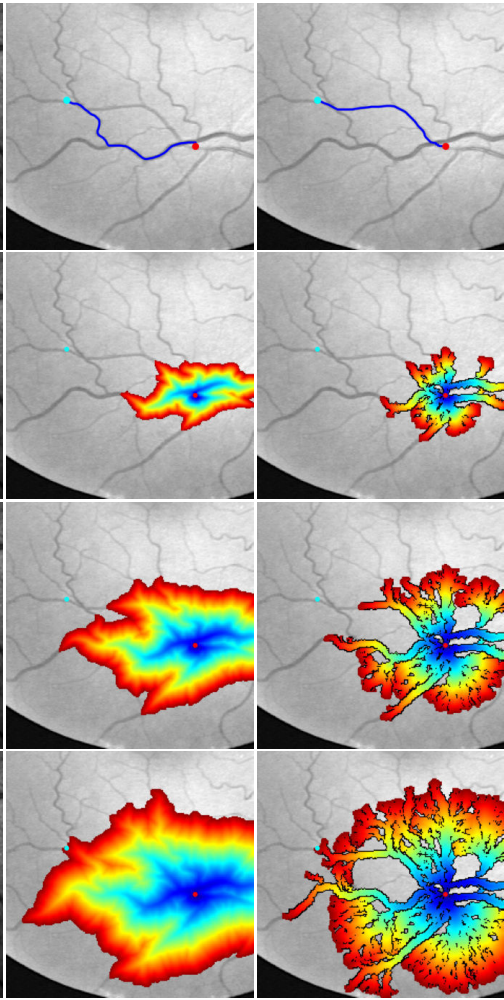

(c) $\operatorname{ArR}$

(d) dArR

Figure 5: The first row shows the detected vessel centerlines (blue lines). The source and end points are respectively denoted by red and cyan dots. The following rows display the course of the fast marching fronts propagation. Columns (a) and (c): Geodesic distance maps derived from the ArR metric [16]. Columns (b) and (d): Geodesic distance maps computed by the proposed dArR metric.

using the projected path $\tilde{\mathcal{G}}_{\mathbf{x}_{\min }, \mathbf{q}}$. In Figs. 4(b) and (c), we illustrate the spatial component $\mathbf{x}_{\min }$ of the latest Trial point $\hat{\mathbf{x}}_{\min }=\left(\mathbf{x}_{\min }, \mathbf{q}\right)$ and the corresponding projected path $\tilde{\mathcal{G}}_{\mathbf{x}_{\min }, \mathbf{q}}$. Moreover, the path in Fig. $4(\mathrm{~b})$ corresponds to a small bending measure is given. If $\mathcal{K}\left(\hat{\mathbf{x}}_{\text {min }}\right) \leq \mathcal{K}_{0}$ which yields $\delta(\hat{\mathbf{x}})=\infty$ such that the criterion is violated and the propagation at $\hat{\mathbf{x}}_{\text {min }}$ should be frozen, see Fig. 4(c) for an example of the large bending measure.

The proposed Riemannian tensor field $\mathcal{M}_{\mathrm{dyn}}$ is updated during the geodesic distance computation which is actually carried out by computing the front- 
freezing indicator $\delta(\mathbf{x}, r)$ in a front advancing procedure as described in Section 3.2. Moreover, we can observe if the propagation should be frozen or not is based on the indicator $\delta(\mathbf{x}, r)$. In implementation, once a point $(\mathbf{x}, r)$ is frozen, all the points at position $\mathbf{x}$ with different radius scale $\left[r_{\min }, r_{\max }\right]$ are frozen and labeled as Accepted. This scheme is helpful to save calculation consuming. It is achieved by defining a new flag map, i.e. a scalar-valued function, $\mathcal{F}: \Omega \rightarrow\{0,1\}$, where $\mathcal{F}(\mathbf{x})=1$ denotes that the point at position $\mathbf{x}$ is frozen. During fast marching fronts propagation, once the latest Trial point $\hat{\mathbf{x}}_{\min }=\left(\mathbf{x}_{\min }, r_{\text {min }}\right)$ on the front is chosen, the $\mathcal{F}\left(\mathbf{x}_{\min }\right)$ value can be checked. If $\mathcal{F}\left(\mathbf{x}_{\min }\right)=1$, the point should be frozen and labeled as Accepted; If $\mathcal{F}\left(\mathbf{x}_{\text {min }}\right)=0$, the local geodesic path $\tilde{\mathcal{C}}_{\hat{\mathbf{x}}_{\text {min }}, \hat{\mathbf{s}}}$ is tracked and the indicator $\delta\left(\hat{\mathbf{x}}_{\text {min }}\right)$ is computed. Then the tensor $\mathcal{M}_{\mathrm{dyn}}\left(\hat{\mathbf{x}}_{\min }\right)$ can be constructed as in Eq. (11) and the geodesic distance value can be estimated. According to the front-freezing indicator $\delta\left(\hat{\mathbf{x}}_{\text {min }}\right)$, if $\mathcal{K} \leq \mathcal{K}_{0}$, the value of $\mathcal{F}\left(\mathbf{x}_{\text {min }}\right)$ is set to 1 which means the front propagation needs to be frozen at $\hat{\mathbf{x}}_{\min }$. The front propagation scheme can be terminated till the end point has been tagged as Accepted or all the grid points have been tagged as Accepted. The pseudo-code for our fast marching-based implementation procedure can be found in Algorithm 1.

The fast marching front propagation process during the computation of the geodesic distance map is displayed in Fig. 5. The geodesic distance map obtained in the radius-lifted space is projected to the domain $\Omega$ by minimizing it along radius dimension as follows:

$$
\mathcal{H}_{\mathbf{s}}(\mathbf{x})=\min _{r \in\left[r_{\min }, r_{\max }\right]} \mathcal{U}_{\hat{\mathbf{s}}}(\mathbf{x}, r)
$$
Fig. 5. Columns (b) and (d) illustrate the geodesic distance maps computed by the our proposed dynamic metric with vesselness map as the scalar function (dArR). The black points in columns (b) and (d) indicate the points which have been frozen due to the violation of the criterion (i.e. $\delta(\cdot)=\infty$ ). The geodesic 225 anisotropic radius-lifted Riemannian metric (ArR) [16] are shown in columns (a) 
and (c). One can point out that the minimal geodesic paths depict the vessels of strong appearances and miss the target vessels. The minimal action maps by the proposed model are shown in columns (b) and (d). Note that the points

can see that the paths obtained by the proposed model can correctly find the target vessels.

\subsection{Discussion}

The proposed method in essence constructs the geodesic metric in a dynamic way and we discuss the differences between our method and two state-of-the-art dynamic minimal path approaches [28, 29].

- In work [28], authors utilize the dynamic isotropic metric established only in the image domain. They introduced a new front freezing fast marching scheme based on the path features which can be detected through the appearance feature of the path or the geometric feature such as the bending measure of the paths. However, This dynamic minimal path approach only invokes isotropic geodesic metrics established in the spatial domain (the image domain), so it cannot benefit from the vessel anisotropy information and also can only detect the vessel centerlines. In order to address the issues mentioned above, we propose a dynamic anisotropic and crossing-adaptive Riemannian metric a in radius-lifted domain based on the geometric features of the local path. As a consequence, the proposed model is able to take advantages of the vessel anisotropy information and also to detect the vessel centerlines and boundaries simultaneously.

- In the work [29], the authors proposed a minimal path model associated with an adaptively Riemannian metric embedded with an appearance feature coherence penalty. The key differences between the model [29] and the proposed model are mainly described as three aspects. Firstly, the method [29] penalizes the geodesic metric by a weighted scalar-valued function derived from the coherence property of the appearance features, 


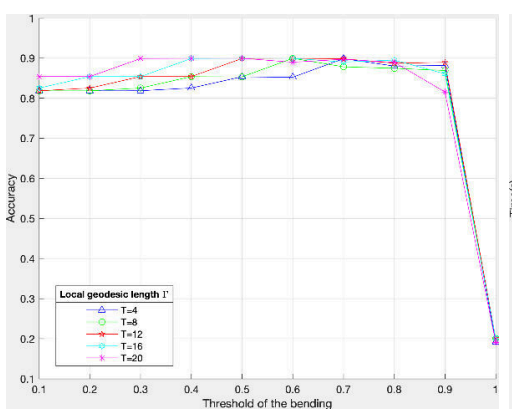

(a)

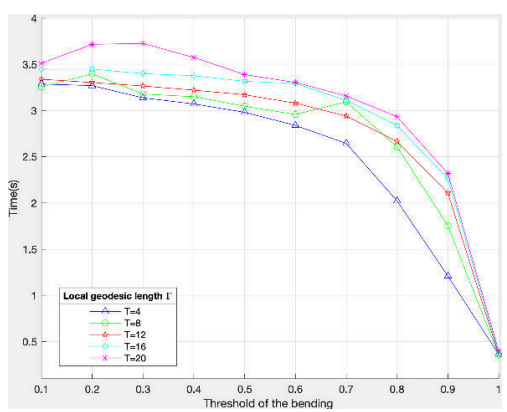

(b)

Figure 6: (a) Segmentation accuracy $\mathcal{R}$ and (b) Running time corresponding to the local geodesic length $\Gamma$ and the bending threshold $\mathcal{K}_{0}$.

instead of freezing the front points. Sometimes one needs to carefully control the importance between the tubular confidence information and the coherence penalization. Secondly, the dynamic metric in [29] is still built in the spatial domain ${ }^{3}$ while our method can detect both centerlines and thickness in one step. Finally, we analyze the implementation difference on the adaptive establishment. In [29], the authors build the dynamic metric using a reference point form a truncated geodesic path vector to distinguish the correct vessel direction in crossing points. In contrast, the basic idea in the the proposed work for the considered crossing-adaptive anisotropy is to reduce or remove the anisotropy property at the crossing points and to keep the anisotropy property at the single vessel points, done by the tool of structure tensor.

\section{Experimental Results and Discussion}

In this section, we conduct the numerical experiments on qualitative and quantitive comparison between state-of-the-art minimal path models and the

\footnotetext{
${ }^{3}$ Indeed, the authors [29] proposed to use one more step to obtain the vessel thickness with a region-constrained minimal path technique.
} 
Table 1: Quantitative comparison results on synthetic images.

\begin{tabular}{c|ccccc}
\hline \multirow{2}{*}{ Method } & \multicolumn{5}{|c}{ Noise level $\delta$} \\
\cline { 2 - 6 } & 0.01 & 0.03 & 0.05 & 0.07 & 0.09 \\
\hline ArR Metric & 0.828 & 0.836 & 0.843 & 0.834 & 0.842 \\
\hline dArR Metric & 0.875 & 0.861 & 0.863 & 0.860 & 0.867 \\
\hline
\end{tabular}

proposed dynamic metrics.

Basic Setting. The front-freezing indicator $\delta$ in Eq. (22) is computed based on the bending measure operator in Eq. (21) of the local truncated geodesic. In numerical implementation, the local geodesic length $\Gamma$ and the bending threshold ${ }_{275} \mathcal{K}_{0}$ should be given. In this work, we conduct an experiment to study the sensitivity of the segmented results to the two crucial parameters $\Gamma$ and $\mathcal{K}_{0}$ on synthetic images, the dataset of which will be described detailed in Section 4.1. The segmentation accuracy and running time are shown in Fig. 6. We set $\Gamma=8$ and $\mathcal{K}_{0}=0.9$ which are reasonable choice for the experiment, since the segmentation accuracy is high and running time is relative small. Besides, the parameter $\epsilon=0.05$ in Eq. (17) is used to determine the anisotropic property of the structure tensor. The parameter $\alpha=5$ in Eq. (16) is related to the influence of the appearance features. The parameter $\beta=0.5$ controls the radius speed. The experiments are performed on a standard Intel Core i5 of $2.7 \mathrm{GHz}$ architecture with 8Gb RAM.

For the proposed dynamic minimal path model, we consider three types of the scalar functions to construct the crossing-adaptive anisotropic radius-lifted Riemannian metrics, respectively relying on the vesselness map (dArR), vessel mask (dArR-M) and vessel skeleton (dArR-S), see Section 3.1. In order to show the advantages of the proposed model in tubular structure extraction, we compare with the ArR metric [16], the variant of the isotropic dynamic minimal path model (dIsoM) [28] and the appearance feature coherence-penalized Riemannian metric with adaptively anisotropy (AFC) [29]. Note that for the 
sake of fair comparison, the dIsoM metric-driven minimal paths are extracted by submitting the scalar-valued function $\mathcal{P}_{\text {scale }}$ defined in Eq. (7) to the geodesic distance propagation scheme with front freezing procedure as depicted in Algorithm 1 .

Evaluation Score. In order to evaluate the proposed dynamic minimal path models quantitatively, we consider an accuracy measurement to validate the segmentation results. Let $\mathcal{S}$ represent the segmentation region from the considered models and $\mathcal{G}$ denotes the region from ground truth data. In addition, $\#\|\cdot\|$ be the number of grid points within the set. Thus the measurement is defined as

$$
\mathcal{R}=\frac{\#\|\mathcal{S} \cap \mathcal{G}\|}{\#\|\mathcal{S}\|} .
$$

One can point out that the measurement $\mathcal{R}$ is ranged within the interval $[0,1]$, where $\mathcal{R}=1$ means that the vessel segmentation is exactly identical to the ground truth. When the measurement $\mathcal{R}$ is used in the vessel centerlines comparison, the ground truth region $\mathcal{G}$ is the dilated centerline from the ground truth by a disk of radius 2 (in grid points) and $\mathcal{S}$ represents the set of grid points located in the detected centerlines.

\subsection{Experiments on Synthetic Images}

In this section, we compare the proposed $\mathrm{dArR}$ metric to the ArR metric [16]. The crossing-adaptive tensor field for this dArR metric is computed by the associated vesselness map. The experiments are conducted on synthetic images in order to study the robustness of the dArR metric. These synthetic images include high curvature lines and crossing lines. All images are blurred by Gaussian noise with 5 different standard deviation levels ranging from 0.01 to 0.09 to evaluate the robustness against noise. Each level of the noise is added independently 3 times. The ground truth is a binary segmentation of all pixels corresponding to the structures.

The segmentation results in terms of the accuracy measurement $\mathcal{R}$ are shown

in Table. 1. The extracted centerlines and boundaries are demonstrated in Fig. 7. From both the qualitative results in Fig. 7 and the quantitative results 


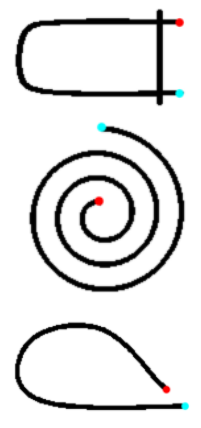

(a) Origin
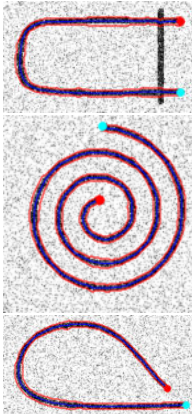

(b) $\delta=0.05$
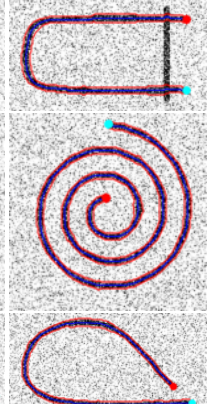

(c) $\delta=0.09$
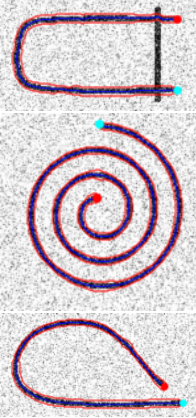

(d) $\delta=0.05$

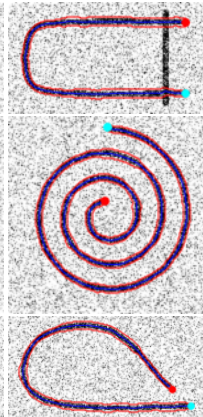

(e) $\delta=0.09$

Figure 7: Boundaries (red lines) and centerlines (blue lines) detected from synthetic images. Source and end points are indicated by red and cyan dots, respectively. (a) Original images. (b) and (c): Segmentation results by the proposed dArR metric. (d) and (e): The segmentation results form the ArR metric.

as described in Table. 1, one can point out that the proposed dArR metric-driven minimal paths are indeed in sensitive to the effects from noise. The minimal paths from the dArR metric can benefit from the use of the front freezing scheme thus can improve the accuracy and robustness of the anisotropic fast marching propagation, leading to favourable centerline and boundary extraction results.

In Fig. 8, we show the minimal paths driven by the dArR metric as well as the AFC metric. In this figure, there are two crossing structures with almost identical gray levels, where the target is longer than another one in the sense of Euclidean curve length [29]. The geodesic path derived using the AFC metric fails to detect the target as shown in Fig. 8(b), since similar gray levels generate similar tubular appearance features. While the minimal paths driven by the dArR metric with bending constraint can freeze the front points which lead to paths of high bending measures, thus the resulting geodesic paths can avoid sharp turning, as shown in Figs. 8(c) to (e). For the AFC metric, a crucial point is that the appearance features of the target vessel should be different to its neighboring ones. Such a requirement may lead to a failure when the situations do not satisfy such a prior. In contrast, the proposed $\mathrm{dArR}$ metric rely on the property from the geodesic paths themselves, instead of the appearance features 


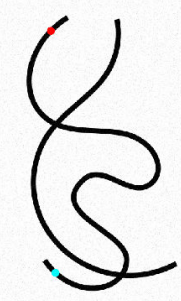

(a)

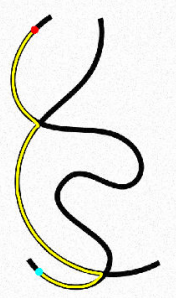

(b)

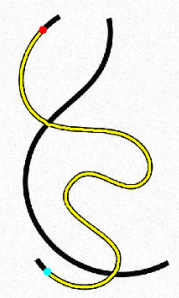

(c)

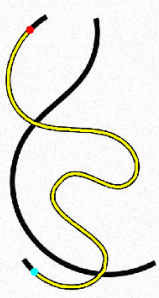

(d)

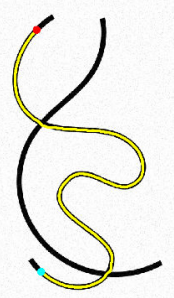

(e)

Figure 8: The minimal paths on synthetic images. (a) The origial images with prescribed points. (b) The minimal path by AFC metric. (c-e): The minimal paths detected by the proposed metric with three different weight function including Vesselness map,Vessel Mask and Vessel Skeleton.

from the image data. Thus in case the target vessels are smooth (without sharp turning), the dArR metric can obtain desired minimal paths.

\subsection{Experiments on Retinal Images}

In this section, we validate the proposed minimal path model on 40 retinal patches obtained from the DRIVE dataset [38] and 40 retinal patches from the IOSTAR dataset [2]. Each patch includes a retinal artery vessel which is near a stronger retinal vein vessel or crosses with it. The average size of the patches from the DRIVE and the IOSTAR is $160 \times 277$ and $223 \times 326$, respectively. Our goal is to extract an artery vessel between two given points. In order to show the advantages of using the crossing-adaptive tensor field, we directly apply the ${ }_{45}$ radius-lifted tensor field $\mathcal{M}_{\text {adap }}$ defined in Eq. (16) for minimal path extraction under a regular anisotropic fast marching algorithm. For simplicity, we refer to $\mathcal{M}_{\text {adap }}$ as caArR in the remaining of this paper.

In Fig. 9, we illustrate the effect of the caArR metric for which the anisotropy is reduced at the crossing points. In column (a), the yellow lines indicate the anisotropy feature vectors of the tubular structures detected by the OOF filter. We can observe that the anisotropy feature vectors located at the cross section are approximately collinear to the directions of the vessel of strong appearance feature. This may lead to incorrect geodesic tangents on these crossing points 


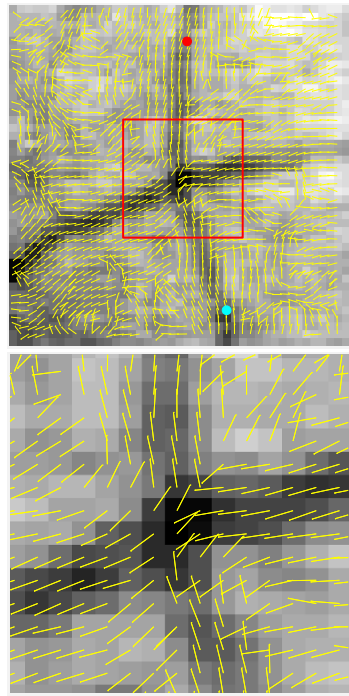

(a)

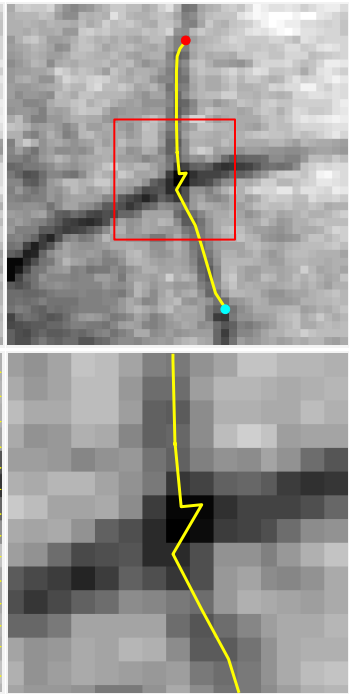

(b)

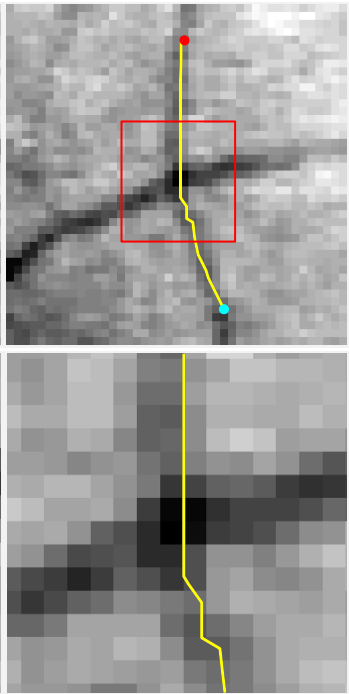

(c)

Figure 9: Qualitative comparison between the ArR and caArR metrics. The geodesic paths are indicated by yellow lines. The source and end points are indicated by red and cyan dots. Column (a): Vessel anisotropy feature vector filed indicated by lines. Columns (b) and (c): Geodesic paths extracted by the ArR and caArR metrics, respectively. The pictures in row 2 respectively illustrate the details in the regions within the red rectangles of Row 1 .

when extract the weak vessel as shown in column (b), where the geodesic path derived by the ArR metric has a bulge in the cross section. Compared with the ArR metric, the minimal path shown in column (c) is smoothed by the caArR metric which has the ability to reduce the anisotropy of the cross section.

In Fig. 10, we compare the caArR metric and the ArR metric on retinal image patches, where the results are shown in columns (b) and (c) respectively. In column (b), we can see that the short branches combination problem occurs. The minimal paths prefer to combine vessel segments with strong appearance features. While in the column (c), the paths obtained from the caArR metric can get the target vessels correctly. Finally, the segmented vessels by the caArR metric are illustrated in column (d).

In Fig. 11, we show the comparison results of the proposed dArR to the ArR metric. The first two rows of Fig. 11 illustrate the minimal path extraction 


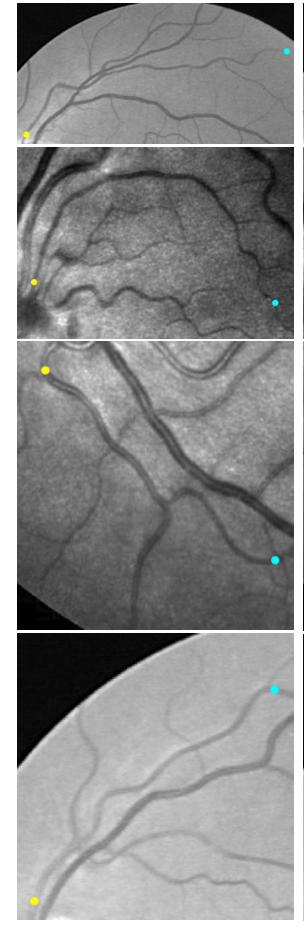

(a)

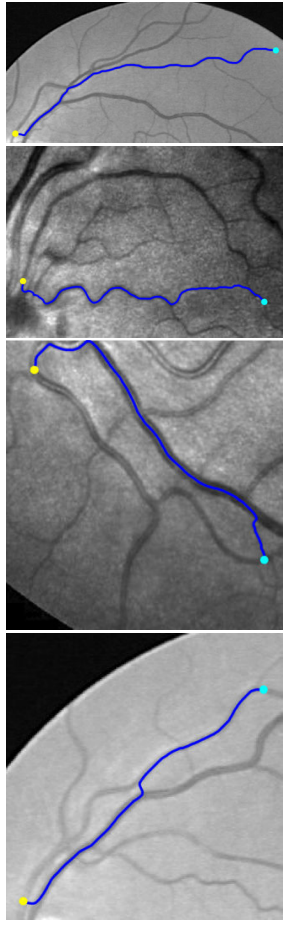

(b)

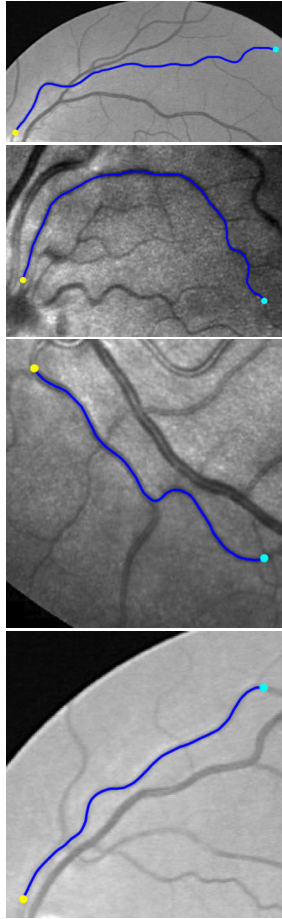

(c)

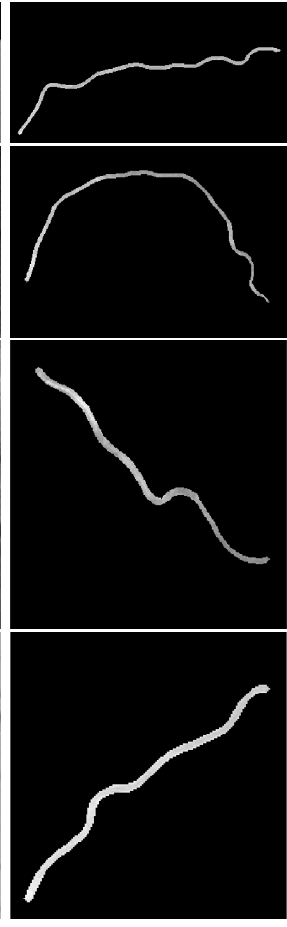

(d)

Figure 10: Qualitative comparison results between the ArR and caArR metrics on retinal image patches. Blue lines represent the centerlines. Yellow and cyan dots represent the source and end points. (a) Patches from retinal images. (b) and (c): Geodesic paths extracted by the ArR and caArR metrics. (d) The segmented vessel regions.

results from the long vessel crossing or near with strong ones. The third row shows the detect results from the image with long and tortuosity vessels. The last two rows denote detected geodesics of vessel regions with strong tortuosity. We observe that the classical ArR metric suffered from short combination problem. In the last two columns, the proposed dArR metric can detect the desired vessel regions by the front frozen scheme.

We quantitatively compare the proposed dynamic models including the dArR, dArR-M and dArR-S metrics, to the existing ArR, dIsoM and AFC metrics on 375 those patches of retina images. The quantitative evaluation is computed with the A-V ground truth by the accuracy measurement $\mathcal{R}$. The segmentation accu- 


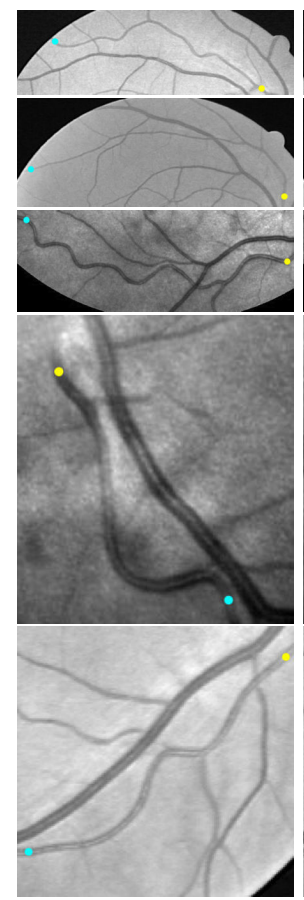

(a)
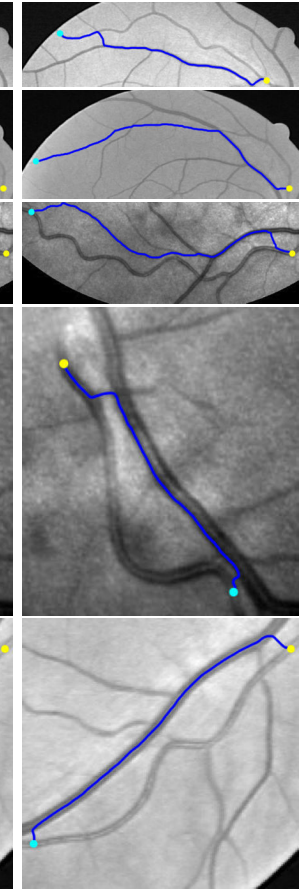

(b)
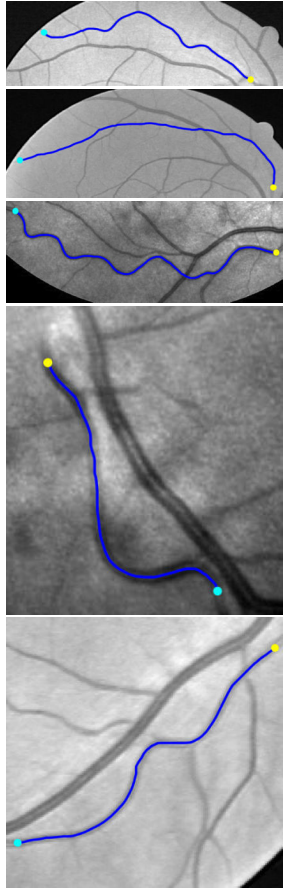

(c)

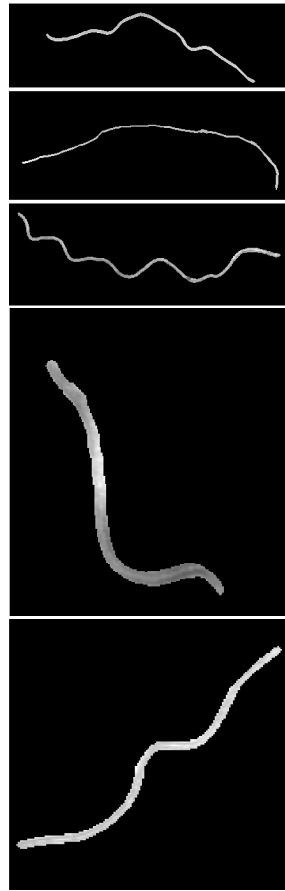

(d)

Figure 11: Qualitative comparison results between the ArR and dArR metrics on retinal images. Blue line represent the centerline. Blue lines represent the centerline. Yellow and cyan dots represent the source and end points. (a) Patches from retinal images. (b) and (c): Geodesic paths extracted by the ArR and dArR metrics. (d) The segmented vessel regions.

racy results for the vessel regions with respect to different metrics are shown in Table. 2. The detection accuracy of the vessel centerlines is shown in Table. 3. From these quantitive results, we can see that the proposed metrics indeed overcomes the shortcuts and short branches combination problems and outperform all the other compared metrics.

The dIsoM ${ }^{4}$ and dArR metrics (also including the dArR-M and dArR-S metrics) are able to take into account the curve bending measure to freeze front points so as to avoid shortcuts and short branches combination problems. From Tables. 2 and 3, we can see that these metrics achieve better vessel segmenta-

\footnotetext{
${ }^{4}$ As mentioned above, dIsoM metric is a variant of the one proposed in [28].
} 


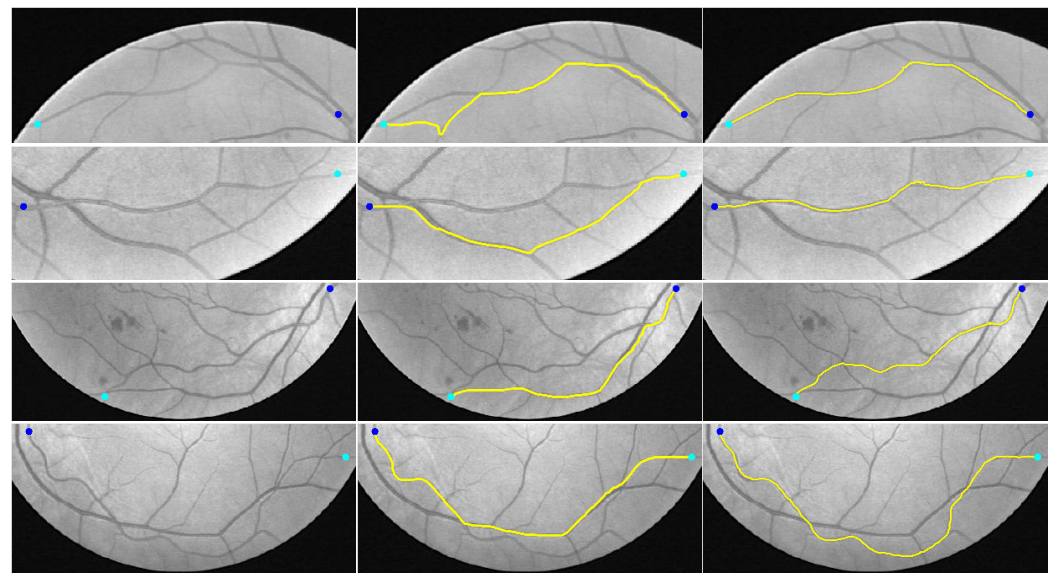

(a)

(b)

(c)

Figure 12: Qualitative comparison results for the AFC and dArR metrics on retinal images. The blue lines represent the extracted geodesic paths. The blue and cyan dots represent the source and end points. (a): Patches from retina images. (b) and (c): Geodesic paths from the AFC [29] and dArR metrics, respectively.

tion results than the static ones on those retinal image patches. However, the proposed metrics differ to dIsoM by encompassing the vessel anisotropy features to build an anisotropic (radius-lifted) Riemannian metric. During the fast marching front propagation and within the vessel regions, the vessel anisotropy features can greatly help the fast marching fronts to propagate faster along the direction of the vessel. As a consequence, in conjunction with the crossingadaptive tensor filed, the performance of the proposed method in the vessel tracking tasks can be improved by the anisotropy enhancement.

In Fig. 12, we show the minimal paths obtained respectively using the AFC metric and the dArR metric. The experimental results are conducted on retina image patches, showing that our proposed dArR metric can detect the correct vessels even if its neighboring or crossing vessels have similar appearance features.

Finally, in Fig. 13, a failure example of vessel extraction for the proposed metric is given, in which parts of the vessel region are mistaken and the vessel centerline is not correct. The vessel directions detected by the OOF do not 


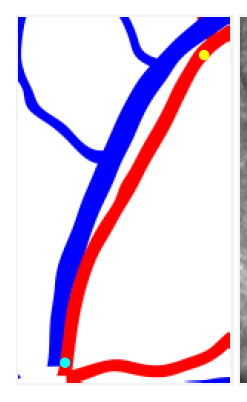

(a)

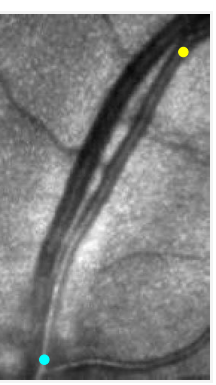

(b)

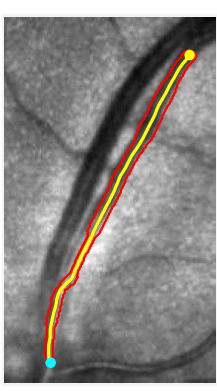

(c)

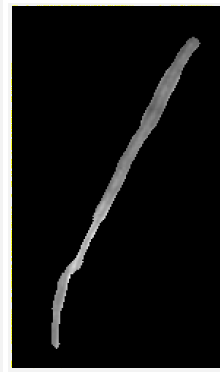

(d)

Figure 13: (a) Ground truth. (b) Image patch with two given points (yellow and cyan dots). (c) The extracted vessel centerline and boundary by the proposed metric. (d) The segmented vessel region.

Table 2: Quantitative comparison results of vessel region segmentation on retinal images from the DRIVE and IOSTAR datasets.

\begin{tabular}{c|ccccc}
\hline Dataset & ArR & caArR & dArR & dArR-M & dArR-S \\
\hline DRIVE & 0.365 & 0.580 & 0.861 & 0.864 & 0.862 \\
\hline IOSTAR & 0.790 & 0.815 & 0.881 & 0.887 & 0.890 \\
\hline
\end{tabular}

agree with the real ones, induced by center reflection. Since the proposed dArR metric is constructed relying on the vessel anisotropy features, the segmentation results in such case fail to get proper results.

We analyze the computation time of each component of the proposed method. There are mainly three steps in the proposed method including the vesselness computation, the crossing-adaptive anisotropic radius-lifted tensor field construction and the fast marching front propagation. The front-freezing indicator is computed during the fast marching front propagation. The results are shown in Table. 4.

\subsection{Experiments on Satellite Images}

In this section, we conduct the experiments on satellite images with rivers, bridges and streets from Google Earth to evaluate the proposed metric. 
Table 3: Quantitative comparison results of vessel centerlines detection on retinal images from the DRIVE and IOSTAR datasets.

\begin{tabular}{c|cccccc}
\hline Dataset & ArR & caArR & dIsoM & dArR & dArR-M & dArR-S \\
\hline DRIVE & 0.425 & 0.557 & 0.803 & 0.849 & 0.842 & 0.850 \\
\hline IOSTAR & 0.683 & 0.730 & 0.832 & 0.862 & 0.859 & 0.865 \\
\hline
\end{tabular}

Table 4: Computation time (in seconds) of each part of the proposed method on retinal images from the DRIVE and IOSTAR datasets.

\begin{tabular}{c|ccc}
\hline Dataset & Vesselness & Metric & FM \\
\hline DRIVE & $1.977 \mathrm{~s}$ & $0.118 \mathrm{~s}$ & $2.080 \mathrm{~s}$ \\
\hline IOSTAR & $3.322 \mathrm{~s}$ & $0.231 \mathrm{~s}$ & $3.146 \mathrm{~s}$ \\
\hline
\end{tabular}

In Fig. 14, we illustrate the qualitative comparison for the ArR metric, the caArR metric and the dArR metric, which are shown from the second to the fourth columns. The first row shows the segmentation results from the image with river crossing several bridges whose intensities are similar to these in the background region. The ArR and caArR metrics-driven minimal paths miss the river direction from the middle located bridge, where the bending of the geodesic path varies strongly. The bending constraint is able to effectively handle this problem. The second row illustrates the segmentation results of a satellite image with two neighbouring rivers and complex background. We aim to detect the smaller one by given two points. We observe that the correct river region cannot be recognized by the ArR metric. The dArR-based minimal paths are capable ${ }_{425}$ of tracking the favourable river correctly without being effected by the stronger river. 


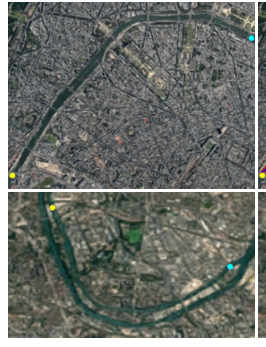

(a)

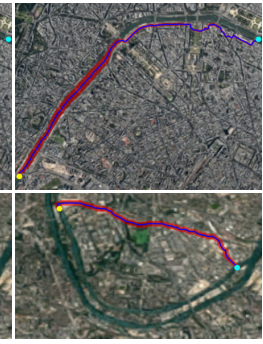

(b)

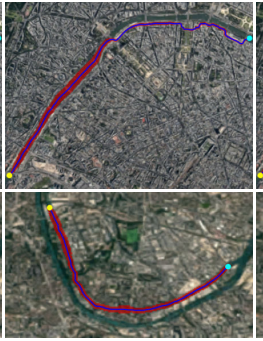

(c)

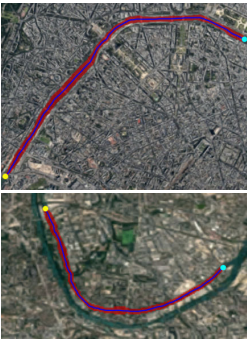

(d)

Figure 14: Qualitative comparison results between the ArR and $\mathrm{dArR}$ metrics on satellite images. The Red region describes the detected road or river and the blue line represents the target centerline. The yellow and cyan dots represent the source and end points. (a) Image patches. (b) to (d): The segmentation results derived from the ArR, caArR and dArR metrics, respectively.

\section{Conclusion}

In this paper, there are mainly three contributions of the proposed method to overcome the shortcut and short branches combination problems in tubular structure detection. Firstly, we propose a crossing-adaptive anisotropic tensor field in the radius-lifted space, which is used to reduce the in favourable effects from the incorrect vessel anisotropy features at the crossing points. This is done by reducing the anisotropy in the crossing points adaptively and simultaneously keeping the high anisotropy in the single vessel points. Secondly, we propose a new way to reduce the influence from the region outside the vessel structure. Finally, we show a way for exploiting front freezing scheme for the fast marching algorithm in a radius-lifted space. We show the practical application of the proposed dynamic minimal path model on synthetic images, retinal images and satellite images. The results demonstrate that our method indeed outperforms classical minimal path methods with static geodesic metrics and the dynamic isotropic metric. In the future work, the proposed method will be extended to $3 \mathrm{D}$ tubular structure detection application and try to reduce the manually intervention. 


\section{Acknowledgments}

We would like to thank the editor and the anonymous referees for their valuable comments, which greatly improved this manuscript. This work was supported in part by the National Key Research and Development Program of China (2017YFC0107903, 2017YFC0109202, 2018ZX10201002-003), by the National Natural Science Foundation of China (Nos. 61902224, 61876037, 61271312, $61401085,31571001,31640028,31400842,61572258$ and 11301074), by the Shortterm Recruitment Program of Foreign Experts (WQ20163200398), by Roche pharma (project AMD_short) and by a grant from the French Agence Nationale de la Recherche ANR-16-RHUS-0004 (RHU TRT_cSVD). The first author thanks China ScholarShip Council (CSC) for the financial support to finish this work.

\section{References}

[1] C. Kirbas, F. Quek, A review of vessel extraction techniques and algorithms, ACM Computing Surveys (CSUR) 36 (2) (2004) 81-121.

[2] J. Zhang, B. Dashtbozorg, E. Bekkers, J. P. Pluim, R. Duits, B. M. ter Haar Romeny, Robust retinal vessel segmentation via locally adaptive derivative frames in orientation scores, IEEE Transactions on Medical Imaging 35 (12) (2016) 2631-2644.

[3] E. Ricci, R. Perfetti, Retinal blood vessel segmentation using line operators and support vector classification, IEEE Transactions on Medical Imaging 26 (10) (2007) 1357-1365.

[4] J. V. Soares, J. J. Leandro, R. M. Cesar, H. F. Jelinek, M. J. Cree, Retinal vessel segmentation using the 2-d gabor wavelet and supervised classification, IEEE Transactions on Medical Imaging 25 (9) (2006) 1214-1222.

[5] A. Sironi, E. Türetken, V. Lepetit, P. Fua, Multiscale centerline detection, IEEE Transactions on Pattern Analysis and Machine Intelligence 38 (7) (2016) 1327-1341. 
[6] C. Zhu, B. Zou, R. Zhao, J. Cui, X. Duan, Z. Chen, Y. Liang, Retinal vessel segmentation in colour fundus images using extreme learning machine, Computerized Medical Imaging and Graphics 55 (2017) 68-77.

[7] N. Armande, P. Montesinos, O. Monga, G. Vaysseix, Thin nets extraction using a multi-scale approach, Computer Vision and Image Understanding 73 (2) (1999) 248-257.

[8] A. Hoover, V. Kouznetsova, M. Goldbaum, Locating blood vessels in retinal images by piecewise threshold probing of a matched filter response, IEEE Transactions on Medical imaging 19 (3) (2000) 203-210.

[9] B. S. Lam, Y. Gao, A. W.-C. Liew, General retinal vessel segmentation using regularization-based multiconcavity modeling, IEEE Transactions on Medical Imaging 29 (7) (2010) 1369-1381.

[10] K. A. Vermeer, F. M. Vos, H. G. Lemij, A. M. Vossepoel, A model based method for retinal blood vessel detection, Computers in Biology and Medicine 34 (3) (2004) 209-219.

[11] E. Bekkers, R. Duits, T. Berendschot, B. ter Haar Romeny, A multiorientation analysis approach to retinal vessel tracking, Journal of Mathematical Imaging and Vision 49 (3) (2014) 583-610.

[12] Y. A. Tolias, S. M. Panas, A fuzzy vessel tracking algorithm for retinal images based on fuzzy clustering, IEEE Transactions on Medical Imaging 17 (2) (1998) 263-273.

[13] L. D. Cohen, R. Kimmel, Global minimum for active contour models: A minimal path approach, International Journal of Computer Vision 24 (1) (1997) 57-78.

[14] T. Deschamps, L. D. Cohen, Fast extraction of minimal paths in 3d images and applications to virtual endoscopy, Medical Image Analysis 5 (4) (2001) 281-299. 
[15] H. Li, A. Yezzi, Vessels as 4-d curves: Global minimal 4-d paths to extract 3-d tubular surfaces and centerlines, IEEE Transactions on Medical Imaging 26 (9) (2007) 1213-1223.

[16] F. Benmansour, L. D. Cohen, Tubular structure segmentation based on minimal path method and anisotropic enhancement, International Journal of Computer Vision 92 (2) (2011) 192-210.

[17] M. Péchaud, R. Keriven, G. Peyré, Extraction of tubular structures over an orientation domain, in: Proceedings of IEEE Conference on Computer Vision and Pattern Recognition (CVPR 2009), 2009, pp. 336-342.

[18] D. Chen, L. D. Cohen, J.-M. Mirebeau, Vessel extraction using anisotropic minimal paths and path score, in: Image Processing (ICIP), 2014 IEEE International Conference on, IEEE, 2014, pp. 1570-1574.

[19] D. Chen, J.-M. Mirebeau, L. D. Cohen, Vessel tree extraction using radiuslifted keypoints searching scheme and anisotropic fast marching method, J. Algorithm Comput. Technol. 10 (4) (2016) 224-234.

[20] Y. Rouchdy, L. D. Cohen, Geodesic voting for the automatic extraction of tree structures. methods and applications, Computer Vision and Image Understanding 117 (10) (2013) 1453-1467.

[21] V. Kaul, A. Yezzi, Y. Tsai, Detecting curves with unknown endpoints and arbitrary topology using minimal paths, IEEE Transactions on Pattern Analysis and Machine Intelligence 34 (10) (2012) 1952-1965.

[22] J. Ulen, P. Strandmark, F. Kahl, Shortest paths with higher-order regularization, IEEE Transactions on Pattern Analysis and Machine Intelligence 37 (12) (2015) 2588-2600.

[23] D. Chen, J.-M. Mirebeau, L. D. Cohen, Global minimum for a finsler elastica minimal path approach, International Journal of Computer Vision 122 (3) (2017) 458-483. 
[24] E. J. Bekkers, D. Chen, J. M. Portegies, Nilpotent approximations of subRiemannian distances for fast perceptual grouping of blood vessels in $2 \mathrm{D}$ and 3D, J. Math. Imag. Vis. 60 (6) (2018) 882-899.

[25] R. Duits, et al., Optimal paths for variants of the 2D and 3D Reeds-Shepp car with applications in image analysis, J. Math. Imag. Vis. 60 (6) (2018) 816-848.

[26] P. Lo, B. van Ginneken, M. de Bruijne, Vessel tree extraction using locally optimal paths, in: Biomedical Imaging: From Nano to Macro, 2010 IEEE International Symposium on, IEEE, 2010, pp. 680-683.

[27] W. Liao, K. Rohr, C.-K. Kang, Z.-H. Cho, S. Wörz, Automatic 3d segmentation and quantification of lenticulostriate arteries from high-resolution 7 tesla mra images, IEEE Transactions on Image Processing 25 (1) (2016) 400-413.

[28] W. Liao, S. Worz, C.-K. Kang, Z.-H. Cho, K. Rohr, Progressive minimal path method for segmentation of $2 \mathrm{~d}$ and $3 \mathrm{~d}$ line structures, IEEE Transactions on Pattern Analysis and Machine Intelligence 40 (3) (2018) 696-709.

[29] D. Chen, J. Zhang, L. D. Cohen, Minimal paths for tubular structure segmentation with coherence penalty and adaptive anisotropy, IEEE Transactions on Image Processing 28 (3) (2019) 1271-1284.

[30] G. J. Parker, C. A. Wheeler-Kingshott, G. J. Barker, Estimating distributed anatomical connectivity using fast marching methods and diffusion tensor imaging, IEEE Transactions on Medical Imaging 21 (5) (2002) $505-512$.

[31] T. Windheuser, T. Schoenemann, D. Cremers, Beyond connecting the dots: A polynomial-time algorithm for segmentation and boundary estimation with imprecise user input, in: Computer Vision, 2009 IEEE 12th International Conference on, IEEE, 2009, pp. 717-722. 
[32] M. Krueger, P. Delmas, G. Gimel'farb, Robust and efficient object segmentation using pseudo-elastica, Pattern Recognition Letters 34 (8) (2013) 833-845.

[33] J.-M. Mirebeau, Anisotropic fast-marching on cartesian grids using lattice basis reduction, SIAM Journal on Numerical Analysis 52 (4) (2014) 15731599.

[34] M. W. Law, A. C. Chung, Three dimensional curvilinear structure detection using optimally oriented flux, in: European conference on computer vision, Springer, 2008, pp. 368-382.

[35] A. F. Frangi, W. J. Niessen, K. L. Vincken, M. A. Viergever, Multiscale vessel enhancement filtering, in: International conference on medical image computing and computer-assisted intervention, Springer, 1998, pp. 130137.

[36] J. Hannink, R. Duits, E. Bekkers, Crossing-preserving multi-scale vesselness, in: International Conference on Medical Image Computing and Computer-Assisted Intervention, Springer, 2014, pp. 603-610.

[37] M. W. Law, G. J. Garvin, S. Tummala, K. Tay, A. E. Leung, S. Li, Gradient competition anisotropy for centerline extraction and segmentation of spinal cords, in: International Conference on Information Processing in Medical Imaging, Springer, 2013, pp. 49-61.

[38] J. Staal, M. D. Abràmoff, M. Niemeijer, M. A. Viergever, B. Van Ginneken, Ridge-based vessel segmentation in color images of the retina, IEEE Transactions on Medical Imaging 23 (4) (2004) 501-509. 